\title{
Probing Cu Diffusion Barrier Layers on Porous Low-Dielectric-Constant Films by Posireonium Annihilation Lifetime Spectroscopy*
}

\author{
HU Yi-Fan(胡一帆 $)^{1 * *}$, SUN Jia-Ning ${ }^{2}$, Gidley D. W. ${ }^{2}$ \\ ${ }^{1}$ Department of Physics, Huazhong University of Science and Technology, Wuhan 430074 \\ ${ }^{2}$ Department of Physics, University of Michigan, Ann Arbor, MI 48109, USA
}

(Received 27 April 2005)

\begin{abstract}
Two kinds of $\mathrm{Cu}$ diffusion barrier layers, sealed films and capped films, on nanoporous low-dielectric-constant films are investigated by positronium annihilation lifetime spectroscopy (PALS). We have found that the minimum thickness of Ta to form an effective diffusion barrier is affected by the pore size. The films with large pores require thick barrier layers to form effective diffusion barriers. In addition, a possible ultra-thin diffusion barrier, i.e. a plasma-induced densification layer, has also been investigated. The PALS data confirm that a porous low-dielectric-constant thin film can be shrunk by exposure to plasma. This shrinkage is confined to a surface layer of collapsed pores and forms a dense layer. The dense layer tends to behave as Ps (positronium) diffusion barriers. Indeed, the controlled thin "skin" layer could prevent $\mathrm{Cu}$ diffusion into the underlying dielectrics.
\end{abstract}

PACS: $68.55 . J k, 78.70 . B j$

Faster response time with a decrease in feature size is among the goals of ongoing improvement in the performance of microelectronic devices. ${ }^{[1]}$ As a result, there have been great efforts to develop ultra-lowdielectric constant thin films as interlayer dielectrics (ILD) to reduce the resistance-capacitance (RC) delay in integrated circuits as the microelectronics industry targets. ${ }^{[2]}$ The integration of copper with porous ILDs has attracted significant interest because the combination will lead to performance improvement in reducing the RC time delay of integrated circuits. However, in most of the promising ILD materials, $\mathrm{Cu}$ is known to have high diffusivity, and the presence of $\mathrm{Cu}$ in the pores of materials with low dielectric constant $k$ could induce some problems including breakdown, cross-talk and current leakage. It is necessary to separate the $\mathrm{Cu}$ interconnect lines from the surrounding ILD by thin liner/barrier layers. ${ }^{[3]}$ A traditional barrier layer was prepared by depositing a thick layer of silicon oxide on the film surface. The advantage of this technique is forming a continuous layer to prevent all $\mathrm{Cu}$ diffusion. However, a thick capped barrier layer can result in electrical property degradation. ${ }^{[4]}$ To solve this problem, Ta sealed barrier layers are developed in recent years. This technique can make the barrier layer very thin, but sometime it is not continuous. On the other hand, Ryan et al..$^{[5]}$ reported unacceptable increases in $k$ accompanied with shrinkage of the low- $k$ film after plasma exposure. Therefore, it is important to understand the correlation among the barrier thickness, continuity and film microstructure such as pore size and distribution in depth. Detecting degradation of electrical properties after integration is straightforward, but cannot reveals the depth-dependent changes in the tiny pores. ${ }^{[6]}$ The simple reason for this is the relative shortage of analytical techniques for characterizing pores, interconnectivity, and $\mathrm{Cu}$ diffusion, all at the $1-10 \mathrm{~nm}$ scale.

In the last several years positronium annihilation lifetime spectroscopy (PALS) has become an established technique for probing nanoporous structure. ${ }^{[7-10]}$ In this technique, a focused monoenergetic beam of positrons is implanted and forms positronium (Ps, the electron-positron bound state) in the film. ${ }^{[7,8]}$ Ps inherently localizes in the pores where its natural lifetime of $142 \mathrm{~ns}$ is reduced by annihilation with molecular electrons during collisions with the pore surface. The reduced lifetime is correlated with void size ${ }^{[7,8]}$ and forms the physical basis for probing pore structure with PALS. The major advantage of depth-profiled PALS is the ability to control the depth distribution of Ps formation by varying the positron beam energy. This is the critical feature that allows analysis of surfaces and the depth-dependent inhomogeneity of the capped or plasma-treated (PT) porous low- $k$ films.

A series of blanket nanoporous low- $k$ films on silicon wafers were prepared. The thin films are processed by spin coating a solution onto a silicon wafer, treating the as-spun film with moist ammonia, and thermally curing the film. For the sealed films and capped film, the initial solution was comprised of hydrogen silsesquioxane (HSQ) resin and two solvents. Methyl propyl ketone, a low boiling solvent, was used to control the thickness of the as-spun film. The second solvent, tetradecane, which has a high boiling point, remained with the resin in the as-spun film. The ratio of the resin to high-boiling point solvent tetradecane is adjusted to control the porosity and hence the dielectric constant of the film (and the av-

* Supported partially by the National Natural Science Foundation of China under Grant No 60476011.

** Email: yifanhu@mail.hust.edu.cn

(C)2005 Chinese Physical Society and IOP Publishing Ltd 
erage pore size). After spin coating, the as-spun film was exposed to moist ammonia to cause the film to gel in the presence of the tetradecane solvent. A hot-plate step of $150^{\circ} \mathrm{C}$ is used to evaporate the low-boilingpoint methyl propyl ketone solvent. The remaining tetradecane was then removed by subsequent heating steps, which include the final cure in the range 400$500^{\circ} \mathrm{C}$. These films are about $1-\mu \mathrm{m}$ thick, with dielectric constant $k$ varying from 1.7 to 2.5 .

A detailed instrument setup and the measurement methods of the PALS technique appear elsewhere. ${ }^{[7,8,11]}$ PALS spectra with $10^{7}$ events were acquired at room temperature with a positron beam and fast lifetime system with a resolution of $\sim 500 \mathrm{ps}$. The Fortran program POSFIT was used for lifetime fitting. According to the preliminary PALS results from the HSQ films, we found that Ps can easily escape into the vacuum if its diffusion length is larger than the film thickness. In order to determine whether the sealed barrier layers are continuous, capped specimen was prepared by uniformly depositing a 100-nmthick layer of silicon oxide on the surface. For sealed films, Ta diffusion barrier layers in thicknesses 5, 15 and $25 \mathrm{~nm}$ were sputter-deposited in Ar with $7 \mathrm{mTorr}$ pressure using an Ener-jet Sputter Coater.

Sealed HSQ films are named for their dielectric value, for example, the sample with $k$ value equal to 2.2 is named LK2.2. Two long-lived Ps components are required for acceptable fitting: one corresponds Ps annihilates in vacuum, and the other corresponds Ps annihilates in film. We believed that vacuum intensities and the film lifetime can be very sensitive to any pinholes or discontinuities in the barrier layer. ${ }^{[11]}$ The $P$ s vacuum intensities at $5.0 \mathrm{keV}$ of the sealed are plotted in Fig. 1. The results of the capped HSQ films are also plotted as the criterion of backscattered Ps intensity in vacuum. Only a small fraction of Ps with the vacuum lifetime $140 \mathrm{~ns}$ is detected, and this is due to backscattered positrons that form Ps at the surface. The intensity of backscattered Ps depends inversely upon the implantation energy. The second component of Ps annihilating in the capped film has an intermediate lifetime, which ranges from 50 to $90 \mathrm{~ns}$ as illustrated in Fig. 2, and corresponds to the mesopores.

Even though only $5 \mathrm{~nm}$ Ta was deposited as the barrier layer, the vacuum intensities of Ps in LK2.2 and LK2.5 films (average pore diameter 3.8 and $2.7 \mathrm{~nm}$, respectively) agree with that of backscattered Ps as determined from the capped films. The Ps lifetimes measured in the mesopores are also consistent with those determined in the capped films. All these results mean that a $5-\mathrm{nm}$ Ta barrier is thick enough to form a continuous layer on LK2.2 and LK2.5 and from Ps lifetime no structural pore change is observed. Thus, we can conclude that the minimum barrier thickness is not more than $5 \mathrm{~nm}$ for these two films.

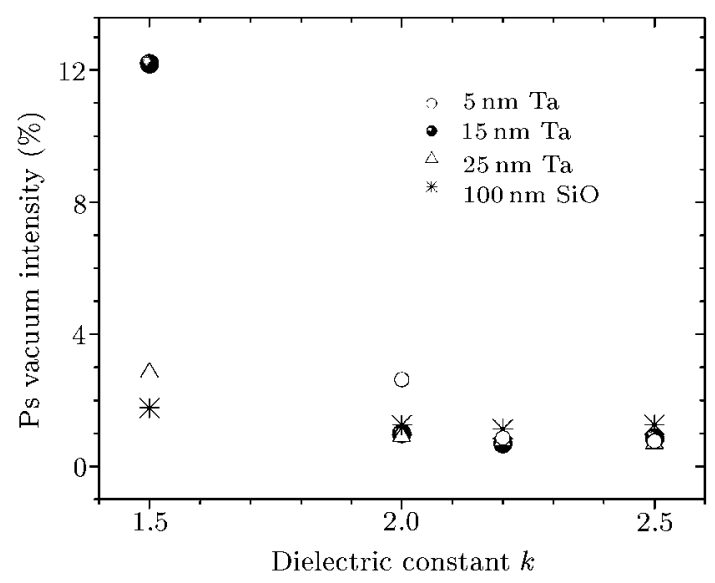

Fig. 1. Ps vacuum intensity in the capped $\left(100 \mathrm{~nm} \mathrm{SiO}_{2}\right)$ and Ta sealed HSQ films at $5.0 \mathrm{keV}$ beam energy.

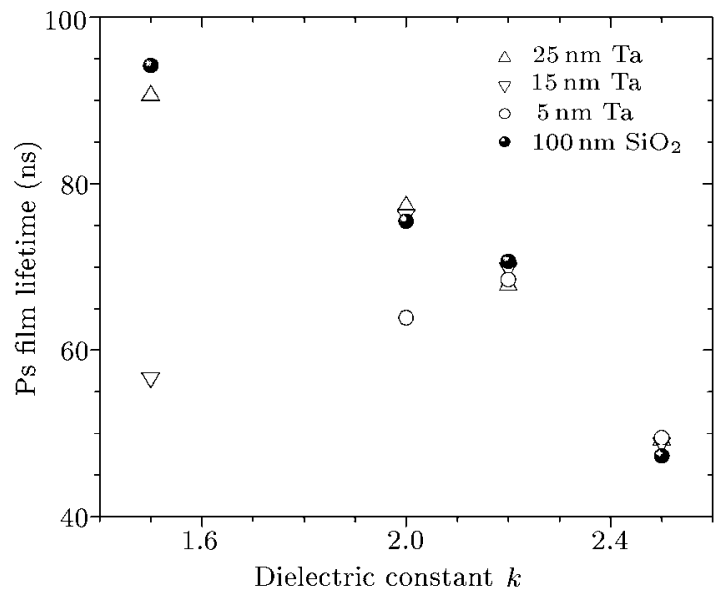

Fig. 2. Fitted Ps film lifetime in the capped and Ta-sealed HSQ films at $5.0 \mathrm{keV}$ beam energy.

However, for the LK2.0 film, a $5 \mathrm{~nm}$-thick-barrier is not completely effective in preventing Ps diffusion out of the film, which has an average pore diameter of $4.5 \mathrm{~nm}$. The Ps vacuum intensity of the LK2.0 film is about twice as high as that expected just from backscattered Ps. The existence of open paths for Ps to escape through the Ta barrier layer is due to some pinholes or discontinuities in the barrier layer. This conclusion can be also confirmed by the reduced fitted Ps film lifetime shown in Fig. 2, because it is generally found that a reduced Ps lifetime is fitted when Ps can escape into vacuum. When the barrier becomes 15 or $25 \mathrm{~nm}$ thick, no escaped Ps is detected and the Ps lifetime is equally as long as that of the oxide capped film. This indicates the formation of a continuous layer, which prevents any Ps atoms from escaping into vacuum despite their many collisions with the barrier. Therefore, we conclude that the minimum Ta barrier thickness for LK2.0 film lies between 5 and $15 \mathrm{~nm}$.

For the 15-nm Ta-sealed LK1.5 film, the vacuum 
intensity of Ps is extraordinarily high. We can conclude that barrier is not continuous on this film, which has the largest pores $(7 \mathrm{~nm})$ of these films. However, when $25 \mathrm{~nm} \mathrm{Ta}$ is deposited onto this film, the vacuum intensity of Ps is only slightly higher than that of backscattered Ps and the Ps film lifetime is slightly lower than that determined in the oxide-capped film. There could be small amounts of Ps leaking into the vacuum through defects in the barrier layer. Although the $25 \mathrm{~nm}$ Ta barrier may still not be perfectly continuous as a diffusion barrier, it is much better than those barriers thinner than $15 \mathrm{~nm}$. Therefore, $25 \mathrm{~nm}$ should be the minimum barrier thickness required for the LK 1.5 film. These results indicate that pore size can affect the minimum barrier thickness.

Unsurprisingly, thick diffusion barriers tend to be required for films with large pores in order to form a continuous and effective barrier layer. Since the pores are highly interconnected, we use an infinitely long cylindrical pore model to deduce pore diameter. According to the extended Tao-Eldrup model, ${ }^{[12]}$ the average pore diameters are determined and plotted in Fig. 3. As can be seen, there is a clear trend between pore sizes and dielectric constant values. High- $k$ and hence low-porosity films tend to have small pores.

On the other hands, systematic plasma treatments of methyl-silsesquioxane (MSQ) film were performed to simulate the processing environment that low$k$ films will encounter during their integration with
$\mathrm{Cu} .{ }^{[5]}$ The blanket MSQ film on a Si wafer has a thickness of $279 \mathrm{~nm}$ and porosity of $58 \%$. Three specimens were exposed to a treatment of nitrogen-based plasma in a commercial plasma enhanced chemical vapour deposition tool at $350^{\circ} \mathrm{C}$ for $10 \mathrm{~s}, 30 \mathrm{~s}$, and $60 \mathrm{~s}$, respectively. A forth film was treated in a commercial Gasonics asher for $10 \mathrm{~s}$ of oxygen plasma ashing. For the MSQ samples, along with a pair of unprocessed nonporous and porous films as control samples, were depth profiled with PALS by varying the implantation energy from 1 to $8 \mathrm{keV} .^{[8]}$

For those PT MSQ films, the second component of Ps annihilating in the film has an intermediate lifetime $\leq 45 \mathrm{~ns}$. The nonporous film has only one long lifetime Ps component, i.e., a small fraction of Ps annihilating in a vacuum as shown in Fig. 4. In the unprocessed porous film, however, much more Ps is detected to annihilate with the vacuum lifetime of $140 \mathrm{~ns}$, indicating that the pores in this film are highly interconnected and open to the vacuum. ${ }^{[8]}$ The fitted intensities of Ps in the vacuum of the PT films and the $\mathrm{O}_{2}$ ashed sample are also illustrated in Fig. 4. All these treated films present a similar intensity of Ps in a vacuum as the unprocessed nonporous film. We believe that there is only backscattered Ps in the vacuum and Ps formed in the film cannot escape into the vacuum in these processed films. Hence, the interconnected pores in the porous MSQ film are sealed after the PT or the ashing process.

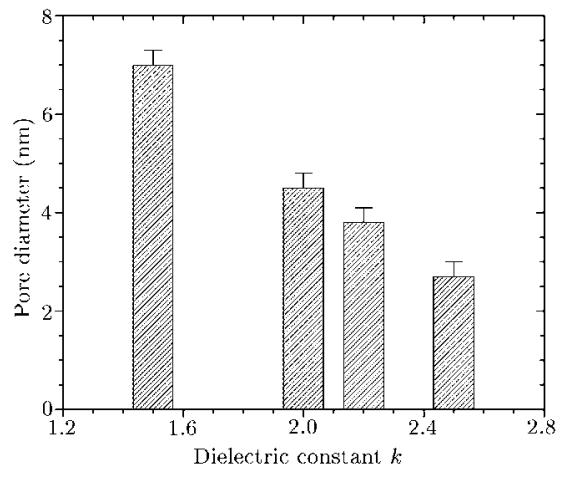

Fig. 3. The relation between pore size and dielectric constant of Ta-sealed HSQ films.

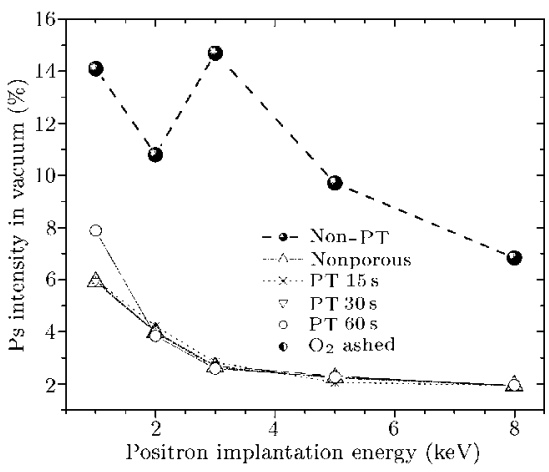

Fig. 4. Ps vacuum intensity in the plasma-treated (PT) and $\mathrm{O}_{2}$ ashed porous films. A unprocessed nonporous film is examined for comparison.

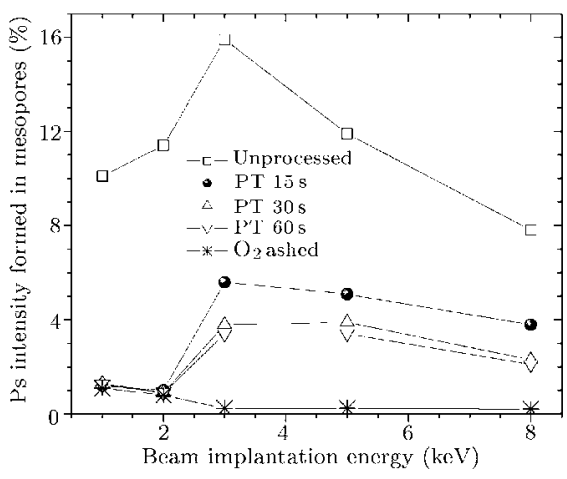

Fig. 5. Ps intensity formed in mesopores (including Ps diffusing into vacuum).
This nonporous layer formed on the surfaces performs effectively as a Ps diffusion barrier, and suppresses Ps formation. Therefore, depth profiling is required to determine the thickness of dense layer. Ps intensity formed in mesopores, which depends on beam implantation energy as shown in Fig. 5. The profiling results show that Ps formation in the mesopores is much weakened in the PT films. In particular, very little Ps annihilates in the mesopores at low implantation energies, where most positrons stop close to the surface. At energies of $3 \mathrm{keV}$ and above, more positrons are able to penetrate the dense layer and to form Ps in the mesopores underneath. A Ps lifetime around $45 \mathrm{~ns}$ is fitted in the mesoporous component, consistent with that observed in the oxidecapped unprocessed film. This indicates that PT processing collapses surface pores without affecting the porous structure underneath. The Ps mesoporous intensity starts to decrease at $5 \mathrm{keV}$ in all the films as a result of the deep implantation of positrons into the 
Si substrate.

There are two possible assumptions for the skin layer formation: The first comes from the pore collapsing and the second comes from pore filling after plasma treatment. We believe that the possibility of the former is larger than that of the latter. The thicknesses of the skin layers have been estimated by making a comparison between the processed films and the unprocessed porous film. For simplicity, we assume that the dense layer is simply formed as a result of the pore collapse and the underneath pore structure has not been affected. Assuming the Ps mesoporous intensity is proportional to the thickness of the mesoporous layer, we can then estimate the thickness of the remaining mesoporous layer by comparing the Ps intensity values in the processed film with that of the unprocessed film. The average thicknesses of skin layer are $24 \pm 5 \mathrm{~nm}, 52 \pm 8 \mathrm{~nm}$, and $90 \pm 5 \mathrm{~nm}$ for the films with $15 \mathrm{~s}, 30 \mathrm{~s}$ and $60 \mathrm{~s}$ PT exposure times, respectively. The ashed film, in particular, shows only negligible Ps signal from the mesopores (less than 0.5\%) throughout the probed energy range of 1 to $8 \mathrm{keV}$. This means that almost all the mesopores have collapsed.

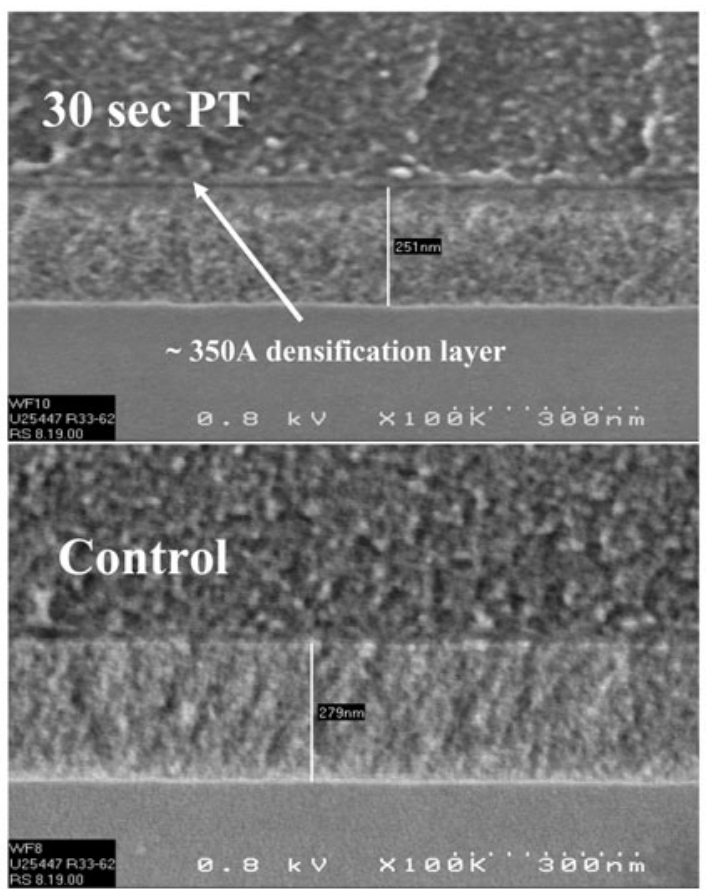

Fig. 6. Cross-sectional SEM image of the nitrogen-based PT 60 s film. A M-bond epoxy layer was deposited on top of the film to facilitate sample preparation.

In order to confirm the collapsing assumption and the estimated thickness, complementary experiments using a Hitachi $5000 \mathrm{H}$ scanning electron microscope (SEM) have been carried out on these films. ${ }^{[4]}$ The SEM results are consistent with the hypothesis based on the PALS results that a skin layer is formed on top of the porous low- $k$ thin film after plasma treatment or ashing process, with the sacrifice of the mesoporosity. The overall thicknesses of the MSQ films are measured in cross-sectional SEM images and are presented along the right-hand side edge of Fig. 5. The original thickness of $279 \mathrm{~nm}$ shrank to $251 \mathrm{~nm}$ after nitrogen based plasma exposure of $30 \mathrm{~s}$. The film is further thinned to $150 \mathrm{~nm}$ in the $\mathrm{O}_{2}$ ashed film, almost the thickness of the dense analog without any mesopores. This clear evidence of film thinning from SEM analysis strongly suggests the pore collapse densification mechanism. These results show that a short time exposure to plasmas can alter the porous structure in low- $k$ films drastically, which will compromise the electrical properties.

In summary, we have studied two kinds of $\mathrm{Cu}$ diffusion barrier layers on porous low- $k$ films with interconnected porosity. These low- $k$ films are made from the same manufacturing procedures with different dielectric constants. Pore size is found to change inversely with the dielectric constant, while the minimum barrier layer thickness has been found to increase with pore size. Depth-profiled PALS has been demonstrated to be a simple and powerful technique in probing integration damage in a porous film with highly interconnected porosity. The presence of a densified surface layer induced by the plasma treatment and ashing process has been detected. No Ps leakage signal into the vacuum is observed in all of the treated films. These over-layers tend to behave as Ps diffusion barriers that could protect the rest of the film from plasma damage. Ideally, these barriers could prevent $\mathrm{Cu}$ diffusion into the underlying dielectrics. Further studies in the future may achieve through the control of the plasma parameters, exposure time, and the low-k film composition.

\section{References}

[1] Peters L 1998 Semiconduct. Int. 2164

[2] Semiconductor Industry Association/USA 1999 International Technology Roadmaps of Semiconductors (San Jose, CA)

[3] Kaloyeros A E and Eisenbraun E 2000 Ann. Rev. Mater. Sci. 30363

[4] Nguyen C V et al 1999 Polymer Prepr. (Am. Chem. Soc. Div. Polym. Chem.) $\mathbf{4 0} 398$

[5] Ryan E T et al 2001 J. Matter. Res. 163335

[6] Morgan M et al 2000 Ann. Rev. Mater. Sci. 30645

[7] Gidley D W et al 2000 Appl. Phys. Lett. 761282

[8] Gidley D W et al 1999 Phys. Rev. B 60 R5157

[9] Petkov M P, Webeer M H, G.Lynn K and Rodbell K P 2000 Appl. Phys.Lett. $\mathbf{7 7} 2470$

[10] Gidley D W et al 2001 New Directions in Antimatter Chemistry and Physics ed Surko C M and Gianturco F A (Dordrecht: Kluwer) p 151

[11] Sun J N et al 2001 J. Appl. Phys. 895138

[12] Dull T L et al 2001 J. Phys. Chem. B 1054567 